\title{
Resection of a giant sternal chondrosarcoma and chest wall reconstruction: a case report
}

\author{
Dahai Liu ${ }^{1,2}$, Zizong Wang ${ }^{1}$, Tong Qiu ${ }^{1}$, Feng Hou ${ }^{3}$, Yi Qin ${ }^{1}$, Yi Shen ${ }^{1}$, Bingxue Song ${ }^{4}$ \\ ${ }^{1}$ Department of Thoracic Surgery, The Affiliated Hospital of Qingdao University, Qingdao, China; ${ }^{2}$ Health Management Center, The Affiliated \\ Hospital of Qingdao University, Qingdao, China; ${ }^{3}$ Department of Pathology, The Affiliated Hospital of Qingdao University, Qingdao, China; \\ ${ }^{4}$ Department of Cardiology, The Affiliated Hospital of Qingdao University, Qingdao, China \\ Correspondence to: Bingxue Song. Department of Cardiology, The Affiliated Hospital of Qingdao University, No. 59, Haier Road, Qingdao 266000, \\ China. Email: bingxuesong0907@126.com.
}

\begin{abstract}
Chondrosarcomas are common bone carcinomas; however, they are uncommon in the sternum, and giant sternal tumors have rarely been reported in advanced-age patients. This study aimed to describe the clinical presentation, method of preoperative planning and surgery, and perioperative management of a giant sternal chondrosarcoma in an advanced-age patient. We describe the case of an 80-year-old woman who presented with a rare giant sternal chondrosarcoma. The patient's symptoms included significant painful swelling and limited activity. The mass was firm and fixed, and the boundary was unclear. We first performed a simulated surgery on a three-dimensional (3D) model using the mimics system for preoperative planning. An extensive resection of the tumor was then performed. Due to the financial status of the patient, the huge chest wall defect was reconstructed with simple ordinary metal locking bone plates and polyester surgical mesh, and good results were achieved. The patient was discharged without any complications 12 days after surgery. The postoperative pathological examination confirmed the diagnosis of primary grade I-II chondrosarcoma. At the 12-month follow-up examination, the patient was completely rehabilitated, and there was no evidence of recurrence. Giant, low-grade sternal chondrosarcoma is an extremely rare disease in elderly women. 3D modeling and simulated surgery are effective approaches for the preoperative planning of surgery. Postoperative ventilators, antibiotics, and nutritional support are also necessary. Using our reconstructive techniques, chest wall reconstruction with polyester patches and orthopedic steel plates could be a safe, reliable and affordable surgery procedure. It may be an appropriate option for similar cases.
\end{abstract}

Keywords: Giant sternal chondrosarcoma; preoperative planning; chest wall reconstruction; perioperative management; case report

Submitted Sep 18, 2021. Accepted for publication Nov 09, 2021.

doi: 10.21037/atm-21-5616

View this article at: https://dx.doi.org/10.21037/atm-21-5616

\section{Introduction}

Chondrosarcoma is a mesenchymal tissue neoplasm that can produce a cartilage matrix $(1,2)$. Chondrosarcoma accounts for $3.5-9 \%$ of primary bone tumors and $20-27 \%$ of primary malignant bone tumors $(1,2)$. Chondrosarcomas are slightly more common in men than women, and the most common locations of chondrosarcomas are the pelvis, ribs, proximal femur, and proximal humerus (1). Most tumors of the chest wall are malignant, and $85-90 \%$ occur in the ribs and
$10-15 \%$ in the sternum. The ratio of chest wall tumors in males to females is 2:1 (3). A giant chondrosarcoma in the sternum is extremely rare in advanced-age patients, and a literature review revealed no previously reported cases (4-7). Yousefi et al. (8) reported a case of huge chest wall tumor at the same location and used bone cement and prolene mesh for chest wall reconstruction. However, the tumor size was much smaller than our case, and the basic condition of the patient was much better. We made more consideration 

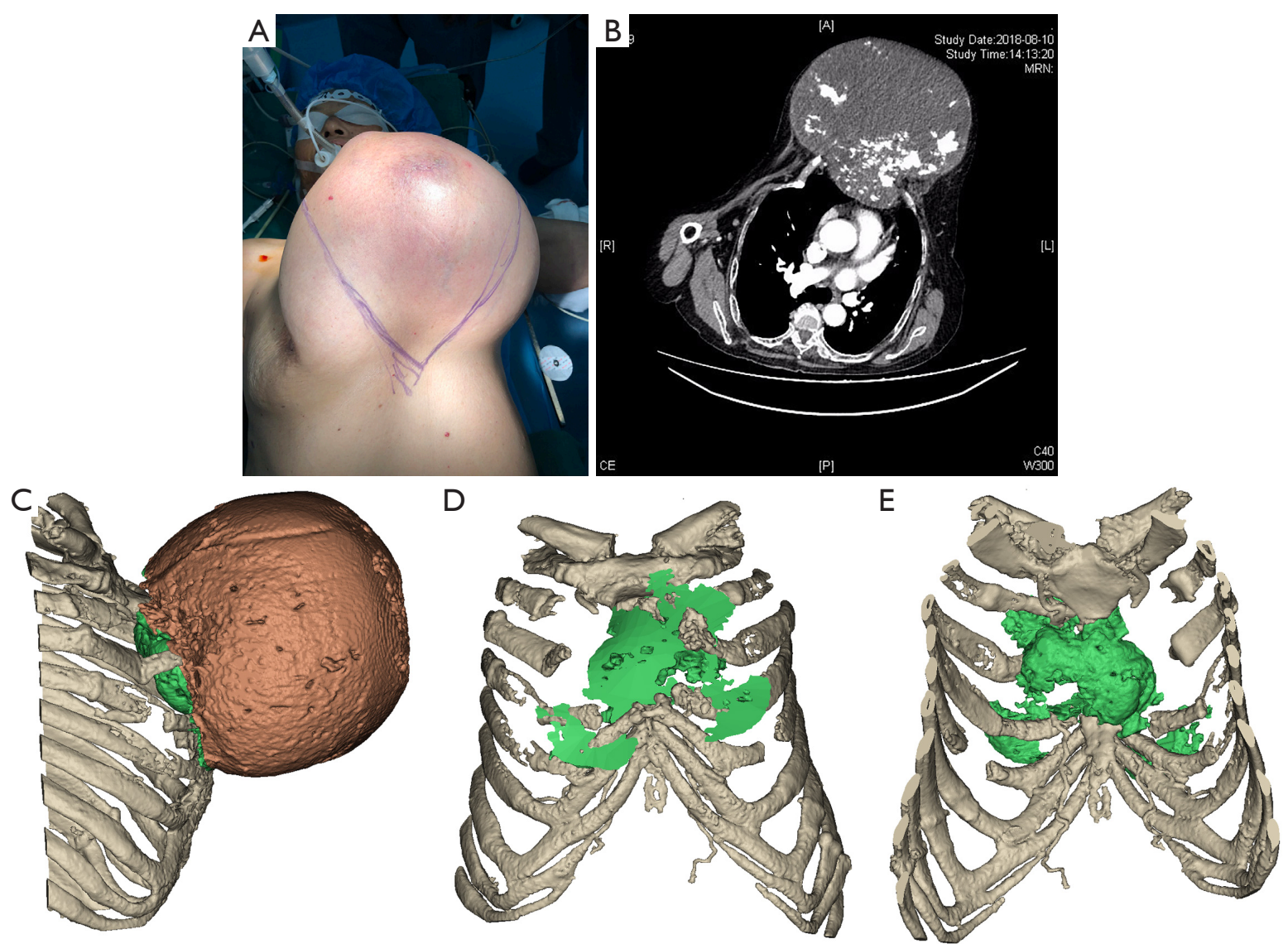

Figure 1 Tumor appearance and CT image 3D reconstruction. (A) A giant tumor in the middle of the anterior chest wall with pressing pain. (B) Enhanced CT of the chest showing a solid mass $\left(25 \times 20 \mathrm{~cm}^{2}\right)$ located in the anterior chest wall associated with the ribs and pericardium. (C) A virtual $3 \mathrm{D}$ model was made by the mimics system for preoperative planning and revealed that the sternum and the bilateral $2^{\text {nd }}, 3^{\text {rd }}, 4^{\text {th }}, 5^{\text {th }}$ costal cartilages and ribs were invaded, and the main blood supply of the tumor was coming from the right internal thoracic artery. (D) The inner view of the basal portion of the tumor. (E) The anterior view of the basal portion of the tumor. CT, computed tomography; $3 \mathrm{D}$, threedimensional.

in preoperative planning and perioperative management. Furthermore, the surgical wound was larger, better drainage was needed to prevent infection and flap necrosis. The huge chest wall defect also needed stronger support to maintain the stability of the thorax. We made improvements in these aspects. An awareness of the treatment and surgical approach for a giant chondrosarcoma in the sternum, especially its perioperative management, is crucial for thoracic surgeons confronted with similar cases.

In this article, we describe an extremely rare case of a giant sternal chondrosarcoma in an advanced-age woman. We present the following case in accordance with the CARE reporting checklist (available at https://dx.doi. org/10.21037/atm-21-5616).

\section{Case presentation}

An 80-year-old female patient presented to our emergency department with significant painful swelling and limited activity associated with a giant protuberant anterior chest wall mass (see Figure 1A). The mass was firm and fixed, and the boundary was unclear. The patient had a previous history of tuberculosis and a negative family history. The tumor was initially found in September 2014, and thereafter continued to grow slowly. The patient had been hospitalized at Qilu Hospital of Shandong University in February 2018, at which time, the tumor was the size of a volleyball, but had ultimately not been treated. The patient had not received any prior treatment before being hospitalized at 
Diagnosis and treatment timeline

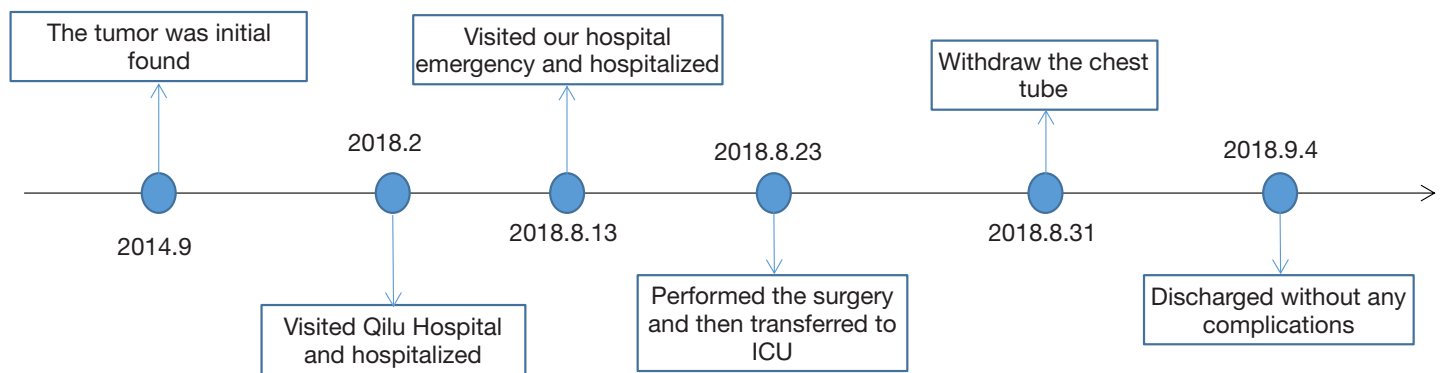

Figure 2 Diagnosis and treatment timeline.

our hospital. Enhanced computed tomography (CT) of the chest revealed a giant mass, which was associated with the sternum, costal cartilage and ribs, and extended to the thoracic cavity and close to the pericardium and measured approximately $25.0 \times 22.0 \times 20.0 \mathrm{~cm}^{3}$ in size on the anterior thoracic wall (see Figure 1B). The tumor had ill-defined margins of the sternum. The patient was diagnosed with a giant sternal tumor and obsolete tuberculosis.

All procedures performed in studies involving human participants were in accordance with the ethical standards of the institutional and/or national research committee(s) and with the Helsinki Declaration (as revised in 2013). Written informed consent was obtained from the patient for publication of this case report and accompanying images. A copy of the written consent is available for review by the editorial office of this journal.

On August 20, 2018, multi-disciplinary treatment (MDT) was administered by the Departments of Thoracic Surgery, Anesthesia, Respiratory and Cardiology. Due to the patient's advanced age, previous tuberculosis history, giant tumor, severe surgical trauma, and other comprehensive factors, the MDT team was of the view that the patient had a high surgical risk and poor prognosis.

We performed a three-dimensional (3D) reconstruction of the chest wall, peritumoral vessels, and the entire tumor, and a virtual 3D model was made by the mimics system to aid in preoperative planning (see Figure 1C-1E). The DICOM file of the patient's enhanced chest CT scan was imported into Mimics Medical 20.0 (Materialise NV, Belgium). The ribs, sternum and enhanced vessels in the chest wall were masked with the predefined thresholds set of the bone (CT, 226 to $1,613 \mathrm{HU})$. The tumor and the surrounded chest wall muscles were masked with a threshold between -5 to $200 \mathrm{HU}$. By using the multiple slice edit function, the tumor was manually separated from the normal soft tissue along its border which could be detected on the CT images clearly. The front of the cartilaginous thoracic cage and the tumor were calculated into the $3 \mathrm{D}$ models from their masks. In order to reveal the extent of the chest wall invasion, the basement of the tumor which invaded the chest wall was then cut off from the main body of the tumor. Thus, the defect of the sternum and the extent of invasion could be comprehensively measured by the surgeons. The $3 \mathrm{D}$ model revealed that the sternum and the bilateral $2^{\text {nd }}, 3^{\text {rd }}$, $4^{\text {th }}$, and $5^{\text {th }}$ costal cartilages and ribs were invaded, and the main blood supply of the tumor was coming from the right internal thoracic artery. The basal portion of the tumor was well defined and clearly demarcated from the surrounding tissue. We performed a simulated surgery on the 3D model. We used the Mimics cutting tool to resect the sternum and ribs at the basal portion of the tumor with maintain a safe margin. Then, the internal thoracic artery was ligated at the upper margin of the second rib. After the tumor was completely removed, we measured the distance of the chest wall margin to the jugular notch and xiphoid process, and the distance of tumor basal portion to ribs margin using a measuring tool. We determined that part of the sternal manubrium and the lower end of the meso-sternum could be retained, and the maximum distance of the chest wall defect was approximately $10 \mathrm{~cm}$ (as measured by the mimics system). Based on the simulated surgery and the patient's financial situation, we decided to use the simplest method to complete the reconstruction of the chest wall. Historical and current information from this episode of care organized as a timeline (see Figure 2).

The surgery was performed on August 23, 2018. The patient was placed in a supine position, and following general anesthesia, a single cavity endotracheal intubation was performed. An extensive fusiform incision of $40 \mathrm{~cm}$ at the center of the tumor was made, and portions of red 

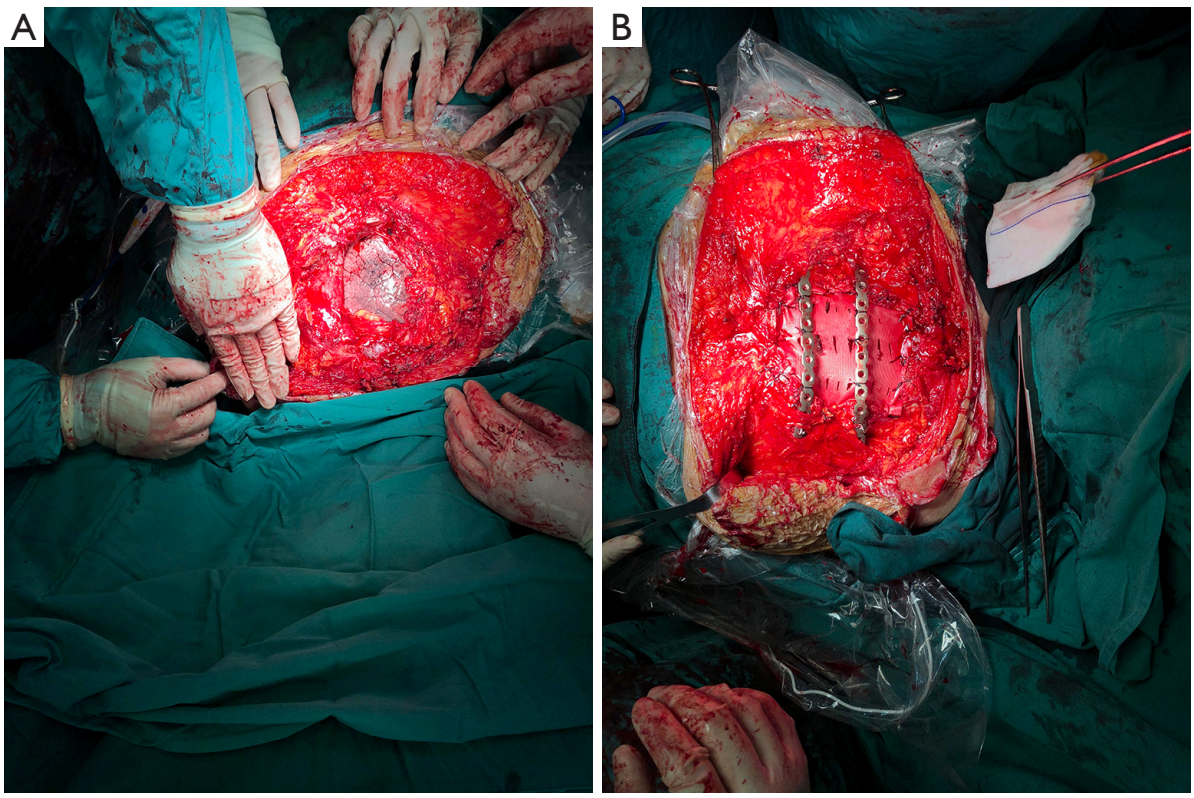

Figure 3 The extent of tumor resection and the method of chest wall reconstruction. (A) The tumor was resected en bloc along with portions of the involved ribs and sternum. (B) The chest wall was reconstructed with two metal locking bone plates and a polyester surgical mesh patch $\left(10 \times 10 \mathrm{~cm}^{2}\right)$.

and swollen skin were resected, and the flap was retained as much as possible for postoperative healing. The tumor was resected en bloc along with most of the sternum, and partial bilateral $2^{\text {nd }}$ to $5^{\text {th }}$ ribs with associated costal cartilage (see Figure $3 A$ ). An intrathoracic drain was inserted in the right pleural cavity. The chest wall was reestablished with two ordinary metal locking bone plates and a surgical polyester patch $\left(10 \times 10 \mathrm{~cm}^{2}\right)$. The polyester patch was sewn to the resection margin of the ribs and sternum to cover the chest wall defect; then, two plates were placed at the defect and perpendicular to the sternum, connected with the $3^{\text {rd }}$ and $4^{\text {th }}$ ribs, and fixed with steel wires (see Figure $3 B$ ). Finally, the flap was sutured with the metal plates and patch, and the incision was sutured layer by layer. The total operating time was $200 \mathrm{~min}$, and the blood loss was 1,200 mL. During the surgery, $700 \mathrm{~mL}$ red blood cells and $400 \mathrm{~mL}$ of plasma were infused. The patient was transferred to the intensive care unit the day after surgery, and back to the general ward the next day. Antibiotics, aerosol inhalation, and nutritional support treatment were administered for 7 days. The chest tube was removed on August 31, 2018, the chest CT was normal. The patient had no discomfort and was discharged without any complications on September 4, 2018. The final diagnosis given by the pathologic examination was low-grade (I-
II) chondrosarcoma (see Figure 4A,4B). Additionally, the pathological examination confirmed that the tumor, which measured $24 \times 22 \times 18 \mathrm{~cm}^{3}$ and weighed $4.5635 \mathrm{~kg}$, and the margins were negative for disease. At the 12 -month followup examination, the patient was completely rehabilitated, and there was no evidence of recurrence.

\section{Discussion}

The diagnosis and treatment of common chest wall tumors are typically straightforward. However, uncommon histological types, sizes, shapes, and locations that do not conform to conventional chest wall tumor types can easily cause diagnostic and therapeutic dilemmas. The sternum is a comparatively unusual location for a chondrosarcoma. Surgical resection remains the only curative treatment for chondrosarcoma because conventional chemotherapy, and radiation therapy are ineffective (5). Based on the simulated surgery, the tumor's nutrient vessel was ligated before removing the tumor, and a part of the sternum was preserved to maintain the stability of the thoracic cage.

The patients came from remote rural areas in China with low education level and relatively lack of medical knowledge. Moreover, the local medical conditions and the economic status were poor. Financial difficulties might have 

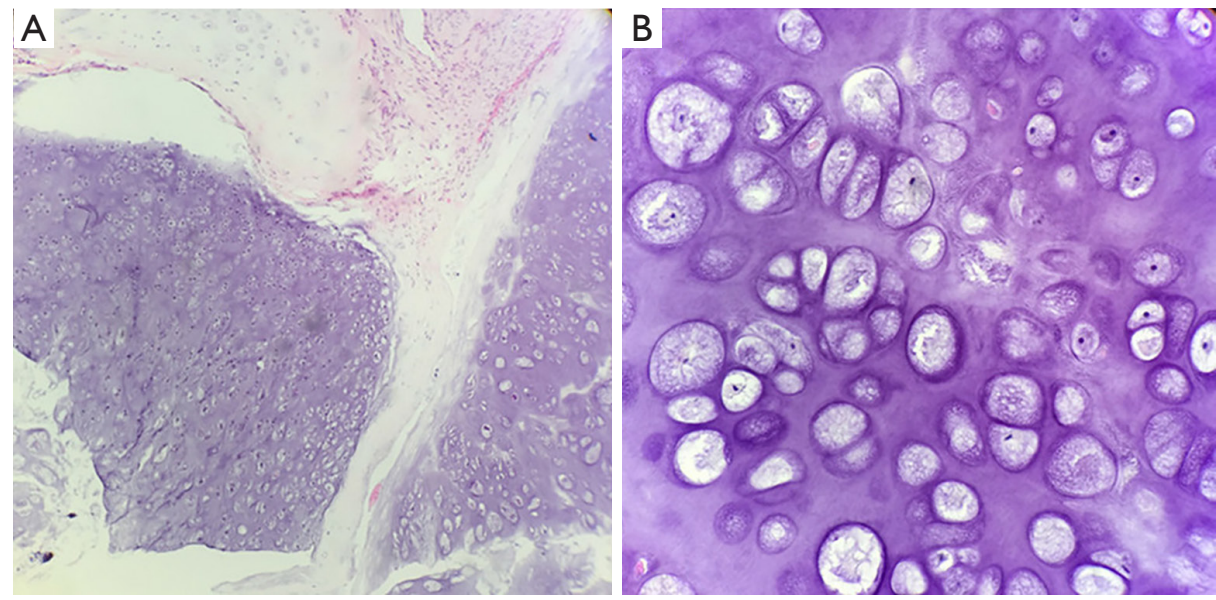

Figure 4 Postoperative pathological diagnosis of the tumor. (A) Pathological specimen showing low-grade chondrosarcoma. The margin of the tumor was unclear and invaded the surrounding soft tissue, as shown by the hematoxylin and eosin staining at $\times 40$ magnification. (B) The nuclei of the tumor varied in size, had a disordered arrangement, and obvious atypia, as shown by the hematoxylin and eosin stain under $\times 400$ magnification.

informed the patient's decision to delay seeking treatment until such a critical stage. The patient was 76 years old at the time of onset, and when the tumor was small, it did not affect her daily life, so she chose not to see a doctor at the early stage of onset. As the tumor grew, her daily activities were affected gradually. When the patient began to see the doctor, many hospitals thought that the surgery opportunity had been lost due to age, tumor size or other reasons and led to further delay in the disease. Finally, the patient came to our hospital for emergency treatment due to severe pain caused by tumor.

There are many methods for reconstructing the chest wall, such as a Teflon patch + titanium alloy plate, bone cement, 3D printing technology, autologous grafting, and a titanium internal fixation system (6-8); however, most of these methods are expensive, technologically dependent, and time consuming. Some surgeons might consider simple chest wall reconstructions to be unsafe and forgo treatment for these patients. In this case, on the basis of the 3D model, we used the most economical and simple method possible to complete the reconstruction of the chest wall and achieved good results. The method of reconstruction was simple, but the technique was crucial for the success of the surgery. Given the patient's large wound surface and excessive exudation, we punched many holes on the patch to improve drainage without weakening the structural strength of the patch. The steel plates were fixed in the margin end of the bilateral rib ends horizontally. The patch and steel plate were fixed to prevent unstable fixation caused by the longterm movement of the ribs. Finally, to enhance the recovery of the flap and avoid necrosis, we sutured the flap together with the steel plate and patch to eliminate the dead space cavity.

Sufficient antibiotics and nutritional support are critical for postoperative recovery in elderly patients with massive trauma. We administered antibiotics for 7 days postoperatively and provided the necessary enteral nutrition in addition to the patient's daily diet. These measures effectively improved the safety of surgery and accelerated postoperative recovery. The patient was satisfied with the treatment due to the comfortable postoperative experience.

Similar to this case, low grade sternal chondrosarcoma does not appear metastasis unlike other malignant tumors, but the local symptoms bring great trouble to patients. As the tumor continues to grow, severe pain, skin ulceration, tumor bleeding, activity limitation and infection could occur, which also affect the patient's prognosis. If the tumor is too large to be completely resected, palliative resection may also benefit these patients, especially elderly patients, who deserve 3-5 years of high quality of life with a safe surgical approach.

Sternal chondrosarcomas similar to the one in this case have rarely been reported, and the exact etiology of such chondrosarcomas is not clear. The cause of the accelerated tumor growth of this case was not clear, and it was unknown whether there was active bleeding in the tumor. Thus, 
further case studies are needed to better understand this rare condition.

In conclusion, giant, low-grade sternal chondrosarcoma is an extremely rare disease in elderly women. The treatment of these giant masses are challenging based on the position, size, and invasive nature of the disease. Complete surgical resection is an optimal treatment for giant sternal chondrosarcoma, and it's necessary to ensure negative surgery margin. Age is not a surgical contraindication for this condition. 3D modeling and simulated surgery are effective approaches for preoperative planning before surgery. Postoperative ventilators, antibiotics, and nutritional support are also necessary. Using our reconstructive techniques, chest wall reconstruction with polyester patches and orthopedic steel plates could be a safe, reliable and affordable surgery procedure. It may be an appropriate option for similar cases.

\section{Acknowledgments}

Funding: None.

\section{Footnote}

Reporting Checklist: The authors have completed the CARE reporting checklist. Available at https://dx.doi. org/10.21037/atm-21-5616

Conflicts of Interest: All authors have completed the ICMJE uniform disclosure form (available at https://dx.doi. org/10.21037/atm-21-5616). The authors have no conflicts of interest to declare.

Ethical Statement: The authors are accountable for all aspects of the work in ensuring that questions related to the accuracy or integrity of any part of the work are appropriately investigated and resolved. All procedures performed in studies involving human participants were in accordance with the ethical standards of the institutional and/or national research committee(s) and with the Helsinki Declaration (as revised in 2013). Written informed consent was obtained from the patient for publication of this case report and accompanying images. A copy of the written consent is available for review by the editorial office of this journal.

Open Access Statement: This is an Open Access article distributed in accordance with the Creative Commons Attribution-NonCommercial-NoDerivs 4.0 International License (CC BY-NC-ND 4.0), which permits the noncommercial replication and distribution of the article with the strict proviso that no changes or edits are made and the original work is properly cited (including links to both the formal publication through the relevant DOI and the license). See: https://creativecommons.org/licenses/by-nc-nd/4.0/.

\section{References}

1. Karpik M, Reszeć J. Low grade chondrosarcoma epidemiology, diagnosis, treatment. Ortop Traumatol Rehabil 2018;20:65-70.

2. Meftah M, Schult P, Henshaw RM. Long-term results of intralesional curettage and cryosurgery for treatment of low-grade chondrosarcoma. J Bone Joint Surg Am 2013;95:1358-64.

3. Allen MS. Chest wall tumors. In: Selke FW, del Nido PJ, Swanson SJ. editors. Sabiston and Spencer's Surgery of the Chest. 8th ed. Philadelphia: Saunders Elsevier, 2010:380-7.

4. He B, Huang Y, Li P, et al. A rare case of primary chondrosarcoma arising from the sternum: A case report. Oncol Lett 2014;8:2233-6.

5. Abraham VJ, Devgarha S, Mathur RM, et al. Dedifferentiated chondrosarcoma of the rib masquerading as a giant chest wall tumor in a teenage girl: an unusual presentation. Korean J Thorac Cardiovasc Surg 2014;47:427-30.

6. Çitak N, Çelikten A, Metin M, et al. Radical resection of a giant recurrent chondrosarcoma of the anterior chest wall. Gen Thorac Cardiovasc Surg 2011;59:512-4.

7. Stanić V, Vulović T, Novaković M, et al. Radical resection of giant chondrosarcoma of the anterior chest wall. Vojnosanit Pregl 2008;65:64-8.

8. Yousefi Y, Sadrizadeh A, Sadrizadeh S. Huge sternal chondrosarcoma: a case report. Asian Cardiovasc Thorac Ann 2018;26:632-4.

(English Language Editor: L. Huleatt)

Cite this article as: Liu D, Wang Z, Qiu T, Hou F, Qin Y, Shen Y, Song B. Resection of a giant sternal chondrosarcoma and chest wall reconstruction: a case report. Ann Transl Med 2021;9(22):1706. doi: 10.21037/atm-21-5616 